Editorial

\title{
Aging of Concrete Structures and Infrastructures: Causes, Consequences, and Cures $\left(C^{3}\right)$
}

\author{
Mohammad Amin Hariri-Ardebili $\mathbb{D}^{1,2}{ }^{1,2}$ Leandro Sanchez, ${ }^{3}$ and Roozbeh Rezakhani ${ }^{4}$ \\ ${ }^{1}$ University of Colorado at Boulder, Boulder, CO, USA \\ ${ }^{2}$ University of Maryland College Park, Park, MD, USA \\ ${ }^{3}$ University of Ottawa, Ottawa, Canada \\ ${ }^{4}$ EPFL, Lausanne, Switzerland \\ Correspondence should be addressed to Mohammad Amin Hariri-Ardebili; mohammad.haririardebili@colorado.edu
}

Received 11 March 2020; Accepted 11 March 2020; Published 7 May 2020

Copyright (c) 2020 Mohammad Amin Hariri-Ardebili et al. This is an open access article distributed under the Creative Commons Attribution License, which permits unrestricted use, distribution, and reproduction in any medium, provided the original work is properly cited.

Aging of any concrete structure is a natural process, but it has become an urgent and critical problem in recent years, during which long-operating dams and nuclear power plants have begun to lose reliable life. A large number of infrastructures all over the world are over 50 years old and suffer from extensive deterioration that affects their serviceability. The high costs associated with preserving the aging structures along with the limited funds allocated for their maintenance pose significant technical and financial challenges, which require the systematic approaches for riskinformed condition assessment. Only in the USA, the American Society of Civil Engineers (ASCE) estimates a required investment amount of about 3.6 trillion dollars by 2020 to improve the condition of infrastructures to an acceptable level. This is more than twice the anticipated available funding level. Aging usually begins to appear in individual elements of the structures, leading to nonuniform or heterogeneous behavior. The most well-known and widespread sign of structural aging is related to weakening of concrete mechanical properties.

Figure 1 schematically presents the performance assessment of concrete structures and infrastructures in their lifetime highlighting the aging effects. Besides typical uncertainties such as uncertainty in environmental loads, several other sources of uncertainty exist, including unknown initial and boundary conditions, unknown damage history to the structure, uncertainties in current laboratory test methods, and finally a big uncertainty in the available predictive models.
In order to develop a comprehensive performance assessment methodology, the past behavior (through diagnosis) should be combined by current observations and tests, and all should be used to predict the future life of the structure. Typically, the hybrid uncertainties are increased with lifetime of the system. Aging and deterioration is a factor which accelerates/intensifies the uncertain response of the structures to imposed environmental actions. Aging and deterioration of concrete structures and infrastructures can be incorporated in both design and analysis phases. In design of new structures, factors such as creep and shrinkage, temperature gradients, sustainability and life cycle cost, and resiliency of the system should be considered. In the analysis of existing structures, material uncertainty and current damage pattern are the key parameters.

The articles presented in this special issue are focused on the state-of-the-art techniques, methods, and applications employed in aging, deterioration, and damage analysis and assessment in concrete structures and infrastructures. Overall, 17 submissions were received by the editorial team, and 8 manuscripts have been accepted for publication.

Freeze-thaw cycling conditions are a primary cause of durability deterioration of concrete structures in the regions with extreme temperature variations. In the paper by Yang et al. "Equation for the Degradation of Uniaxial Compression Stress of Concrete due to Freeze-Thaw Damage," the authors conducted a series of experiments on concrete specimen and determined the freeze-thaw-based damage variable. Subsequently, they proposed an equation for the 


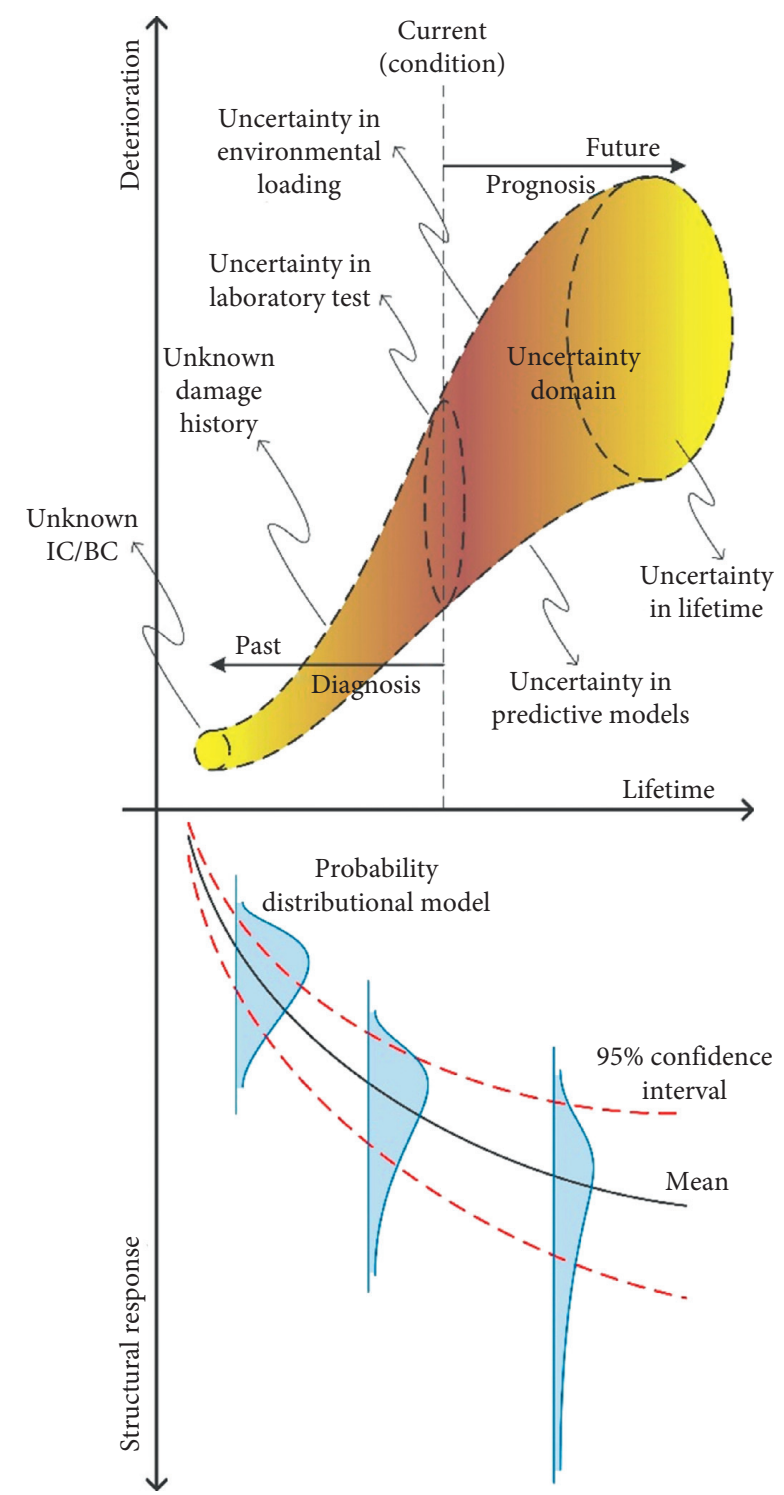

Figure 1: Diagnosis and prognosis of deteriorating concrete structures incorporating various uncertainties.

stress-strain constitutive relation including the freeze-thaw damage variable. They pointed out the observed changes in the elastic modulus with increase in the freeze-thaw cycle number.

Concrete paving stones are fabricated by mixing cement, aggregate, water, and additives in certain ratios. They are widely used in urban road, pavement, and recreation areas. Therefore, increasing their durability and strength is the key factor in sustainability design. In the paper by Bakis "Increasing the Durability and Freeze-Thaw Strength of Concrete Paving Stones Produced from Ahlat Stone Powder and Marble Powder by Special Curing Method," the author proposed a method to use construction waste materials, i.e., Ahlat stone powder and marble powder, in fabricating interlocked paving stones. Both the durability and freezethaw strength of the produced material increased by the special curing method.
Sulfate attack on cement is one of the reasons for degradation of concrete durability and subsequently reduces the service life of the concrete structures. In the paper by Liu et al. "Research on Sulfate Attack Mechanism of Cement Concrete Based on Chemical Thermodynamics," the authors characterized the relationship between temperature and the Gibbs free energy of erosion products generated during the sulfate attack on cement. The proposed model, which was based on principles of chemical thermodynamics, determined the phase composition, microstructure, crystal form, and morphology of erosion products before and after sulfate attack. They pointed out that the sulfate attack has double effects on mechanical properties of specimens.

Accurate performance evaluation of expressway pavement is a vital factor to determine the pavement design scheme and the future maintenance program. In the paper by Yang et al. "Highway Performance Evaluation Index in Semiarid Climate Region Based on Fuzzy Mathematics," the authors proposed a method based on fuzzy mathematics in order to evaluate the performance of a case study pavement. They incorporated multiple sources of fuzziness and randomness in their calculations. The performance grade is quantified by an iterative scheme and contrasted with traditional methods.

Although the long-span concrete girder bridges (including continuous rigid-frame bridges) have been widely used in construction, they suffer from excessive long term deflection. In the paper by Niu and Tang "Effect of Shear Creep on Long-Term Deformation Analysis of Long-Span Concrete Girder Bridge," the authors developed a systematic framework for long-term creep calculation of girder bridges using commercial finite element package. Based on linear creep and the superposition principle, the proposed method can consider both shear creep and segmental multiage concrete effect. For a case study bridge, they reported that shear creep causes $10 \%+$ in long-term deformation. However, the impact of shear creep is close for a bridge with different degrees of prestressing.

The posttensioning by monostrands in substitute cable ducts is a highly efficient method for strengthening of existing bridges in order to increase their load-bearing capacities in terms of current traffic load and to extend their service life. In the paper by Svoboda et al. "Strengthening and Rehabilitation of U-Shaped RC Bridges Using Substitute Cable Ducts," the authors described strengthening and rehabilitation of 100+-year-old bridges. The proposed method minimizes the interventions into the constructions, unseen method of cable arrangement, and the absence of impact on appearance.

Adjacent precast concrete box-beam bridges have been a popular solution for small- and medium-span bridges worldwide. Although nonlinear FEA provides an accurate redundancy assessment of box-beam segments, its application is not always feasible for practitioners. In the paper by Leng et al. "Structural Redundancy Assessment of Adjacent Precast Concrete Box-Beam Bridges in Service," the authors proposed a simplified approach based on linear FEA coupled with field load testing to address the particular structural feature and topology of adjacent precast concrete box-beam 
bridges. This method reduces the computation complexity and improves the reliability.

Multihazard resilience and sustainability of the structures decrease by aging of their components. In the paper by Hayashi et al. "Fundamental Investigation on Seismic Retrofitting Method of Aging Concrete Structural Wall using Carbon Fiber Sheet-Constitutive Law of Rectangular Section," the authors developed a method for seismic strengthening of aging RC buildings by wrapping the structural members with carbon fiber sheets. According to a series of monotonic uniaxial compression tests, they found that the compressive strength decreases and the ultimate strain increases as the ratio of long to short side of the rectangular cross section increases. They also proposed evaluation formulas for the constitutive law of concrete elements with rectangular cross sections.

We hope that this special issue would shed light on the recent advances and developments in the area of aging concrete structures and infrastructures and attract attention by the scientific community to pursue further research and studies on causes, consequences, and cures $\left(\mathrm{C}^{3}\right)$ of aging and deteriorating concrete components in various scales (micro, meso, and macro).

\section{Conflicts of Interest}

The editors declare that they have no conflicts of interest regarding the publication of this Special Issue.

\section{Acknowledgments}

The authors would like to express our appreciation to all authors for their informative contributions and the reviewers for their support and constructive critiques that made this special journal issue possible. The authors also appreciate the contributions by Dr. Alain Giorla (Guest Editor).

Mohammad Amin Hariri-Ardebili Leandro Sanchez Roozbeh Rezakhani 